\title{
Spatiotemporal patterns of catch and discards of the Israeli Mediterranean trawl fishery in the early 1990s: ecological and conservation perspectives
}

\author{
DOR EDELIST ${ }^{1}$, OREN SONIN ${ }^{1,2}$, DANIEL GOLANI ${ }^{3}$, GIL RILOV $^{4}$ \\ and EHUD SPANIER ${ }^{1}$ \\ ${ }^{1}$ Department of Maritime Civilizations and The Leon Recanati Institute for Maritime Studies, The Leon H. Charney \\ School for Marine Sciences, Faculty of Natural Sciences, University of Haifa, Mount Carmel, Haifa 31905, Israel. \\ E-mail: blackreefs@gmail.com \\ ${ }^{2}$ Ministry of Agriculture and Rural Development, Department of Fisheries and Aquaculture The Agricultural Center P.O. \\ Box 30, Beit Dagan 50250, Israel. \\ ${ }^{3}$ Department of Evolution, Systematics and Ecology, The Hebrew University of Jerusalem, 91904 Jerusalem, Israel. \\ ${ }^{4}$ Israel Oceanographic and Limnological Research. Tel- Shikmona, P.O.B. 8030, Haifa 31080, Israel.
}

SUMMARY: The spatiotemporal patterns of discards and catch composition of Israeli trawlers were examined using a 4-year (1990-1994), 324 haul dataset. Haul depth was found to be the main grouping variable for hauls, although significant seasonal differences were also found. $28.3 \%$ of the total catch was discarded, and there was a $40.1 \%$ discard percentage in shallow hauls. According to these figures, annual discards for the Israeli trawl fleet for the study period are estimated at ca. 440 to $700 \mathrm{t}$. Both the biomass and the number of discarded specimens peaked in summer, as well as the percentage of juvenile fish of commercial species. These findings suggest that a summer moratorium on trawling would reduce discards. The percentage of specimens of Indo-Pacific origin decreased from $51 \%$ in depths shallower than $37 \mathrm{~m}$ to $24 \%$ between 38 and $73 \mathrm{~m}$, and $8 \%$ in deeper strata. Discards along the Israeli coast were comparable to those observed elsewhere in the Mediterranean. The findings presented here are the first quantitative account of fish community assemblages in the nearshore waters of the easternmost part of the Mediterranean, and thus provide valuable information for comparisons with more current datasets that are currently being assembled.

Keywords: bottom-trawl, Israel, discards, catch composition, Lessepsian migration, fishery management.

RESUMEN: PATRONES ESPACIO-TEMPORALES DE LA CAPTURA Y LOS DESCARTES DE PESQUERÍA DE ARRASTRE MEDITERRÁNEA ISRAELÍ AL INICIO DE LA DÉCADA DE LOS NOVENTA: PERSPECTIVAS ECOLÓGICAS Y DE CONSERVACIÓN. - Se analizaron los patrones espacio-temporales de los descartes y la composición de la captura de la flota de arrastre de Israel utilizando datos de 4 años (1990-1994), 324 caladas. La profundidad resultó ser el principal factor en la agrupación de las caladas, si bien se encontraron asimismo diferencias significativas entre estaciones. Se descartó el $28.3 \%$ de la captura total, alcanzándose el $40.1 \%$ en las caladas realizadas en las aguas más someras. Según estos porcentajes, se estimó una captura anual descartada por la flota de Israel en ese período de 440-700 t. La biomasa, el número de ejemplares descartados, así como el porcentaje de juveniles de especies comerciales fue mayor en verano. Estos resultados sugieren que una veda de arrastre en verano favorecería la disminución de la captura descartada. El porcentaje de ejemplares de origen indo-pacífico descendió desde $51 \%$ en aguas de una profundidad inferior a $37 \mathrm{~m}$, al $24 \%$ entre $38-73 \mathrm{~m}$ y al $8 \%$ en el estrato más profundo. Los descartes en la costa israelí fueron similares a los observados en otras zonas del Mediterráneo. Se presentan por primera vez resultados cuantitativos relativos a comunidades de las aguas costeras del extremo oriental del Mediterráneo, aportándose información para su comparación con datos más recientes que en la actualidad están siendo obtenidos.

Palabras clave: arrastre de fondo, Israel, descartes, composición de la captura, migración lessepsiana, gestión de pesquerías. 


\section{INTRODUCTION}

It is considered that marine communities have been altered by multiple human stressors. One of the most influential of these stressors is fishing, specifically bottom trawling (Alverson et al., 1994, Hall et al., 2000, Jackson et al., 2001, Pauli et al., 2002). Trawling was criticized for its low selectivity and damaging effects on the benthic habitat as early as the 18th century (Sacchi, 2008). Low selectivity leads to large portions of the catch being discarded at sea due to the species being unmarketable or too small. These discards are an important measure of the impacts of fishing on marine stocks (Alverson et al., 1994). The present study deals with discards, rather than bycatch. It argues that bycatch may be a misleading term for the spatially and temporally heterogeneous multi-species Mediterranean trawl fishery. Whereas bycatch is a loosely defined term, discards are unanimously defined as the part of the catch which is brought onto the deck and then thrown back into the sea (Alverson et al., 1994, Hall et al., 2000, Tsagarakis et al., 2008).

Alverson et al. (1994) did not specifically address discards in the Mediterranean; however, Kelleher (2005) assigned the Mediterranean and Black Sea fisheries (excluding the Levant) a mere $4.9 \%$ discard ratio based on an analysis of $24 \%$ of the total catch. Davies et al. (2009) took a more thorough approach and estimated Mediterranean discards at 306000 tons, or $21.1 \%$ of the total catch. $85 \%$ of these discards originated in the trawl fisheries, which were estimated to have a $45-50 \%$ discard ratio. Various other studies (Stergiou et al., 1998, Machias et al., 2001, D’Onghia et al., 2003, Sánchez et al., 2004, 2007) have assigned discard ratios of $20-50 \%$ to various trawl fisheries in the western and central Mediterranean. El-Mor et al. (2002) and Alsayes et al. (2009), however, found that only $15-25 \%$ of the catch was discarded in Egypt. Recently, in two extreme cases, local discards were found to exceed $70 \%$ in Portugal (Esmeralda Costa et al., 2008) and Turkey (Soykan and Kincigil, 2006). Discard practices vary annually and seasonally throughout the Mediterranean (Machias et al., 2001, Lleonart and Maynou, 2003, Sánchez et al., 2004). For some commercial species, this is because fishermen adjust the actual minimum landing size to match market demand rather than to comply with the Minimum Landing Size (MLS) regulations. For other species (most notably goatfishes) this means that juvenile specimens are consistently landed and marketed. MLS regulations in Israel have been arbitrarily set for several prominent commercial species but are not enforced.

Depth and fishing season were found to be the major factors contributing to the discard rate and catch composition in many Mediterranean trawl fisheries (Stergiou et al., 1998, Machias et al., 2001, D’Onghia et al., 2003, Sánchez et al., 2004, 2007). These findings have been used to justify and manage seasonal trawl closures and minimal trawling depth limitations. The time of day was also shown to have significant effects on catch composition, but mostly as a confounding factor with depth (Wassenberg et al., 1997). There are also spatial differences between different trawling zones. In order to understand these, it is important to determine the characteristics of and variation in local fishing practices (Sánchez et al., 2007).

The Levantine ecosystem differs from the rest of the Mediterranean in phenomena such as Levantine Nanism, in which organisms are smaller and reproduce earlier. This phenomenon was hypothesized by Por (1989) and demonstrated by Sonin et al., 2007 for the red mullet Mullus barbatus. The Levant is also consistently invaded by Indo-Pacific species (e.g. Spanier and Galil, 1991, Rilov and Galil, 2009). This bioinvasion is particularly reshaping the shallow shelf fauna, mainly due to the shallowness of the main invasion vector the Suez Canal (Rilov and Galil, 2009).

The present study analyzes catch, discard and assemblage composition data for the Israeli trawl fishery for the years 1990-1994. This analysis is the first quantitative study of Israeli trawl catches and the first to describe the spatio-temporal patterns of the demersal fish community in the Levant. It focuses on the proportion of discarded specimens, a measure which has thus far been overlooked in Mediterranean trawl catch studies, most of which focus on discarded biomass alone. From a conservation perspective, these data are vital for an understanding of the rates of change vectors and how they are reflected through the fishery. They can serve as a baseline for future comparisons of the status of fish and fisheries in the rapidly changing eastern Mediterranean, and as such assist in ecosystem-based management of the trawl fishery.

\section{MATERIALS AND METHODS}

\section{Description of the fishing fleet, study area and typical catch and effort}

Israeli trawling fleets operate along $170 \mathrm{~km}$ of coastline, up to $20 \mathrm{~km}$ offshore. Some additional, smallscale activity took place near the coast of northern Sinai until the mid 1990s. Trawling occurs in two main geographic areas (Fig. 1), and the same fishing gear is used in both areas. South of Haifa, trawling lanes run parallel to the shore and maintain the haul depth along the isobaths. The wide, shallow continental shelf of southern Israel means that more shrimp trawling, generally performed between 20 and $40 \mathrm{~m}$, is carried out than in the northern fields. The narrower northern shelf and curvature of Haifa Bay often dictate curved or semi-circular trawling lanes. The primary target fish species have traditionally included high priced species such as prawns, mullids and groupers, common sea breams (such as bouge and pandoras) and lizard fish. Hake are also targeted, but at greater depths (Snovsky and Shapiro, 1997). 


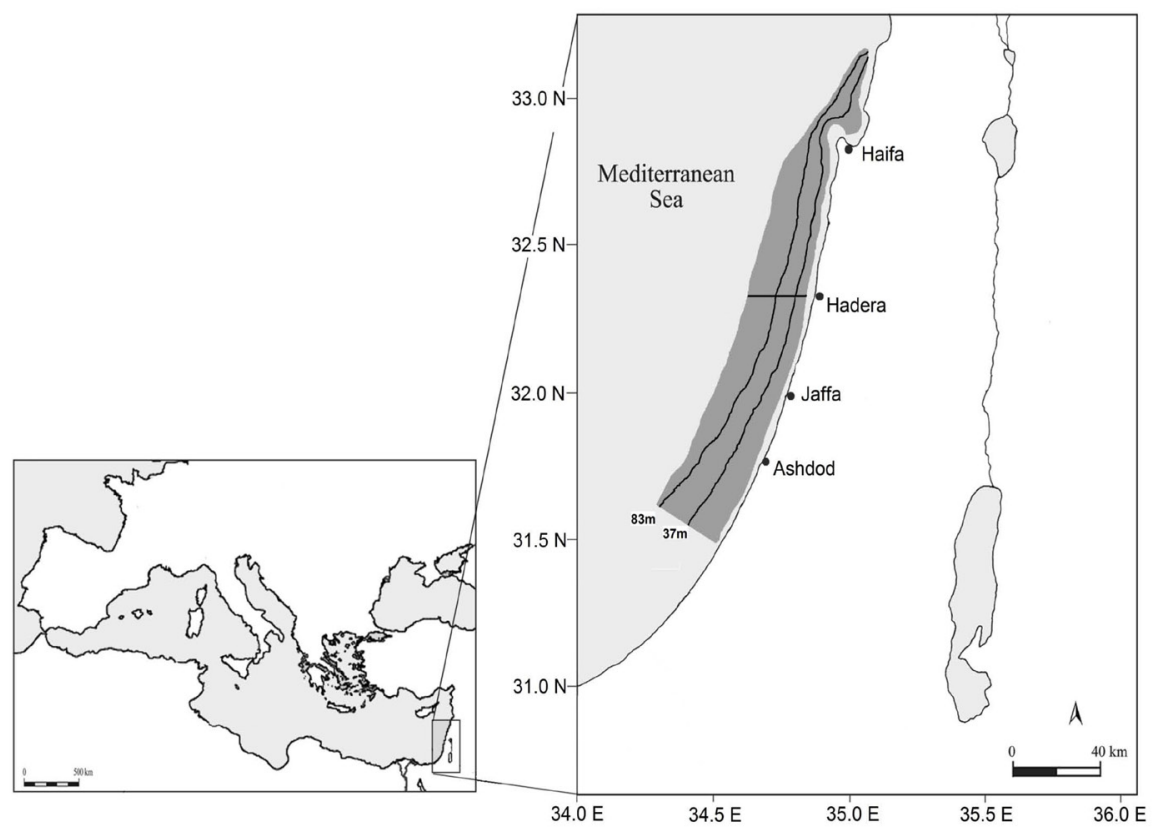

FIG. 1. - Map of the study site, with the main locations and isobaths mentioned in the text. The dark grey area represents the study area, covering all the trawling grounds on the Israeli coast in the study period.

The present study refers to data collected in the early 1990s, when 30-34 vessels, each with a mean engine power of 214-228 hp, were spending a total of 5152-6214 days at sea each year (Snovsky and Shapiro, 1997). The fleet size was frozen at 30 licenses in 1995 and today only 23 out of the 30 vessels are active, possibly due to rising fuel prices and declining catches. This fleet size limit has not proven effective in halting declines in CPUE (catch per unit effort; Fig. 2). A 45day summer trawling moratorium was established in 1998-99, but the policy was discontinued the following year despite encouraging interim results, including increasing catches and larger specimens of commercial species (Pisanty et al., 2000). Currently, the fleet trawls all year round, spending only ca. 4000 days/year at sea. It is important to note that this reduction in fishing effort is mitigated by an increase in mean engine power (currently $294 \mathrm{hp}$ ), which allows increased effort per sea-day. Before 2004-5, the fleet trawled for hake in the spring, and also for rose shrimps Parapenaeus longirostris and red shrimps Aristeus antennatus and Aristeomorpha foliacea. In recent years stocks have dwindled, and now the fleet operates almost exclusively on the continental shelf between $15 \mathrm{~m}$ (the minimum depth allowed by law) and $150 \mathrm{~m}$. This trend towards shallower trawls is in opposition to the efforts in other Mediterranean fisheries, for which regulation of shallow trawling has led to the development of deep-sea trawl fisheries (D'Onghia et al., 2003, Esmeralda-Costa et al., 2008). Such spatial changes in fishing effort alter species compositions, and therefore more Lessepsian migrants are caught by trawlers fishing in the shallows (see Results). The gear has changed very little since 1990 , and 40-48mm (at the cod end) diamond-mesh nets

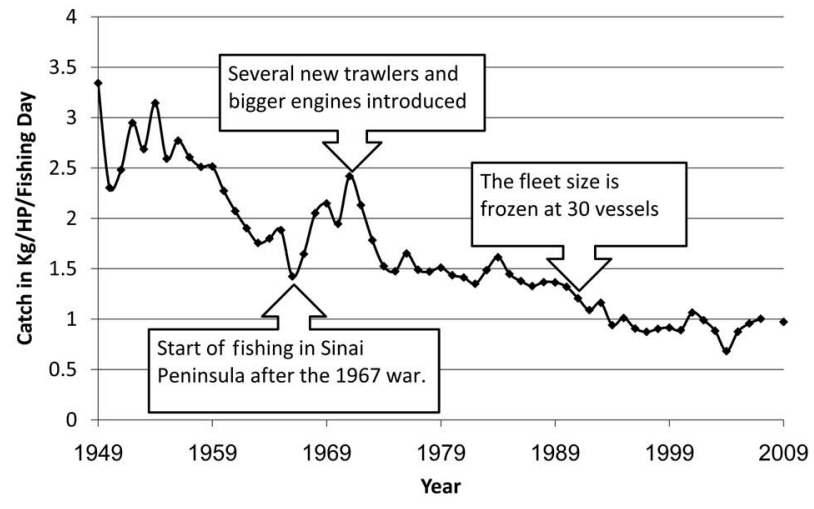

FIG. 2. - Declines in CPUE (catch per unit effort) of Israeli trawlers based on data collected by the Department of Fisheries in the Ministry of Agriculture since 1948. The effort unit used was engine horsepower per day at sea, as used by Garcia and Demetropoulos (1986).

are still used by all trawlers. Annual catches in the early 1990s fluctuated between 1107 and $1757 \mathrm{t}$, with a mean of $1409 \mathrm{t}$; in the last 3 years, catches did not exceed $1227 \mathrm{t}$ per year. While catches of hake, grouper, goatfish and meagre have sharply declined in recent decades (Scheinin, 2010), increases were noted for Chondrichthyes and for the invasive tiger prawn Marsupenaeus japonicus. Since shrimp trawling is more fuel efficient, today almost all vessels target $M$. japonicus in the shallows (15-40 $\mathrm{m})$ at night.

\section{Data collection}

Data were collected from 324 commercial trawl hauls carried out between April 1990 and December 1994 by the Israel Fishery Department. These surveys 
took place onboard trawlers fishing the Israeli continental shelf, between latitudes $31^{\circ} 20^{\prime} \mathrm{N}$ and $33^{\circ} 05^{\prime}$ $\mathrm{N}$. Depths ranged between 15 and $300 \mathrm{~m}$. The gross, discarded and landed catch weights were estimated for 173 out of the 324 hauls in which two or more of these variables were recorded, and therefore total catch analysis was possible. A sample of ca. 5\% of the biomass $(2.7-12.5 \mathrm{~kg}$ with a mean of $5.5 \pm 2.4 \mathrm{~kg}$ per haul) was extracted from each haul. While this partial-sampling method may miss rare species, it allows nearly all the fauna in the nets to be properly sampled and the time-consuming length measurements to be carried out onboard. Fish were taxonomically identified to species level, except for several cases in which only the genus was noted. Fish Total Length (TL), cephalopod Mantle Length (ML) and crustacean Carapace Length (CL for crabs, eye to telson length for shrimps) were measured to the closest $0.5 \mathrm{~cm}$ interval. The discard lengths defined in the present study are described in Table 1 and were subsequently used to differentiate between the two discarded fractions: non-commercial species and juveniles of commercial species. These lengths were determined empirically, i.e. they were estimated based on observations of onboard sorting practices, fishermen's experience and minimum landing size (MLS), rather than on the actual onboard sorting of specimens, which may vary considerably due to factors such as season, abundance, and market considerations (Machias et al., 2001, Demestre et al., 2008).

\section{Data analysis}

Four factors (season, depth, time of the day and geographical trawling area) were tested for their effects (Wilcoxon signed rank test, $\mathrm{P}<0.05$ ) on discard proportions of both the biomass (expressed in $\mathrm{kg}$ per hour) and number of discarded fish, which were classified as either undersized commercial specimens or non-commercial species. Other variables examined included mean species richness (S), Shannon (H') and Simpson diversity and evenness (Pielou's J) as well as the mean proportion of Lessepsian migrants in hauls.

Seasons were defined as four equal three-month periods. The time of day was defined as either day or night. Depths of hauls were divided into 3 depth strata. The $37 \mathrm{~m}$ isobath was chosen to separate the shallow from medium depth due to bottom type: sandy to $37 \mathrm{~m}$ and muddy in deeper strata. This depth is the median point of the 25-50 m sand-mud transition zone described by Sandler and Herut (2000). The $83 \mathrm{~m}$ isobath was chosen as the transition point between medium and deep strata because it approximates the median depth for hauls deeper than $37 \mathrm{~m}$. The two study areas, north of Hadera vs. south of Hadera, span the entire trawling range of the Israeli fleet. Though the two areas are adjacent, they were analyzed independently in this study because they are trawled by two different groups of trawlers (which use similar fishing techniques). Haifa-based trawlers operate north of Hadera, while fields south of Hadera are mostly fished by trawlers that dock in Jaffa and Ashdod (see Fig. 1). We compare the northern and southern areas to test our hypothesis of a higher Lessepsian migrant abundance in the south.

Catch composition analysis was performed using the PRIMER-E v6 statistical software package (Clarke and Warwick, 2001) as follows: For clustering of hauls and Multi Dimensional Scaling analysis (MDS), data was $\log \mathrm{x}+1$ transformed (to allow for the expression of less common species in the assemblage) and then ordered in a Bray-Curtis similarity matrix. The PERMANOVA routine in PRIMER was used to test the significance of differences and the interactions between factors. This application tests the simultaneous response of variables to factors in an ANOVA design using permutations. The SIMPER routine in PRIMER was used to identify the most dominant species in each stratum, defined as the species which contributed most to the Bray-Curtis similarity. To this end, sample data was square-root transformed in order to weight the more abundant species (e.g. Clarke and Warwick, 2001).

\section{RESULTS}

A total of 145 species (or genera, where specific identification was uncertain) belonging to 75 families was identified in surveys (Table 1). Of 124 bony fish species, Sparidae (17 species) and Carangidae (8 species) were the most dominant families. Seven cartilaginous fish species, 10 crustacean species and 6 species/ genera of cephalopods were recorded. Sixty-two species were consistently discarded, 52 were commercial, and 40 were assigned a minor commercial value due to their scarcity, low price or both (Table 1). Bony fish accounted for $69.4 \%$ of the total number of specimens. Crustaceans, despite being represented by only 10 species, accounted for $28.3 \%$ of all specimens. The total landed biomass harvest rate was $19.7 \mathrm{~kg} \mathrm{~h}^{-1}$, while 7.74 $\mathrm{kg} \mathrm{h}^{-1}$ were discarded, culminating in a total discard mean per-haul of $28.2 \%$ of the biomass. Of all the sampled specimens, $46.7 \%$ were discarded. For bony fishes only, this figure was slightly higher at $52.3 \%$ (Table 1).

All four factors were found to have significant effects (Wilcoxon, $\mathrm{P}<0.05$ ) on the discarded proportion of the catch, and the factors 'depth' and 'season' generally had lower p values. Depth and season also had significant effects on Shannon's H', Pielou's J and Simpson's D, as well as species richness. The time of day and geographic area did not however have significant effects on evenness.

The highest seasonal biomass discard ratio was recorded in summer (38.6\%; Fig. 3a) and almost doubled that of spring or winter. It coincided with a very high summer proportion of discarded specimens (61\% of bony fish; Fig. 4a). Furthermore, during summer $74 \%$ of these were undersized juveniles of commercial species. In no other season did any of the last two parameters exceed 52\%. Shannon's diversity and species richness significantly decreased from means of 
TABLE 1. - List of species recorded in surveys, their origin, commercial value and abundance in 324 trawl samples, as well as their landed and discarded fractions in the catch. N, number of specimens; Minor, minor commercial value; Com., commercial; Disc., discarded; * Lessepsian migrant; ** discard size was set according to MLS regulations; *** species were not separated in samples and were recorded as one taxa.

\begin{tabular}{|c|c|c|c|c|c|c|}
\hline Species & Family & Value & $\mathrm{N}$ & $\begin{array}{l}\text { Discard } \\
\text { Size in } \mathrm{mm}\end{array}$ & $\begin{array}{c}\text { No. } \\
\text { Landed }\end{array}$ & Discard \% \\
\hline \multicolumn{7}{|l|}{ Bony Fishes } \\
\hline Pagellus erythrinus & Sparidae & Com. & 7284 & $110^{* *}$ & 1918 & 73.6 \\
\hline Upeneus moluccensis* & Mullidae & Com. & 6230 & 90 & 4524 & 27.3 \\
\hline Boops boops & Sparidae & Com. & 5469 & 130 & 1979 & 63.8 \\
\hline Mullus barbatus & Mullidae & Com. & 4774 & 90 & 3624 & 24.1 \\
\hline Callionymus filamentosus* & Callionymidae & Disc. & 3631 & - & 0 & 100 \\
\hline Leiognathus kluzingeri* & Leiognathidae & Disc. & 3569 & - & 0 & 100 \\
\hline Mullus surmuletus & Mullidae & Com. & 3501 & 90 & 2525 & 27.9 \\
\hline Spicara smaris & Centracanthidae & Minor & 2491 & 130 & 139 & 94.4 \\
\hline Pagellus acarne & Sparidae & Com. & 2483 & 120 & 1402 & 43.5 \\
\hline Dentex macrophthalmus & Sparidae & Com. & 2093 & 110 & 328 & 84.3 \\
\hline Saurida undosquamis* & Synodontidae & Com. & 1796 & $160 * *$ & 1289 & 28.2 \\
\hline Trachurus mediterraneus & Carangidae & Com. & 1795 & 130 & 833 & 53.5 \\
\hline Engraulis encrasicolus & Engraulidae & Minor & 1573 & 110 & 25 & 98.4 \\
\hline Lepidotrigla cavillone & Triglidae & Disc. & 1525 & - & 0 & 100 \\
\hline Merluccius merluccius & Merlucciidae & Com. & 1090 & 140 & 849 & 22.1 \\
\hline Macrorhamphosus scolopax & Macrorhamphosidae & Disc. & 838 & - & 0 & 100 \\
\hline Sardina pilchardus & Clupeidae & Minor & 764 & 110 & 200 & 73.8 \\
\hline Lithognathus mormyrus & Sparidae & Com. & 763 & $110 * *$ & 546 & 28.4 \\
\hline Citharus linguatula & Citharidae & Minor & 731 & 150 & 86 & 88.2 \\
\hline Alepes djedaba* & Carangidae & Com. & 729 & $110 * *$ & 547 & 24.9 \\
\hline Serranus hepatus & Serranidae & Disc. & 600 & - & 0 & 100 \\
\hline Upeneus pori* & Mullidae & Com. & 583 & 90 & 481 & 17.5 \\
\hline Sphyraena chrysotaenia* & Sphyraenidae & Com. & 515 & 150 & 494 & 4.1 \\
\hline Trachinus draco & Trachinidae & Disc. & 453 & - & 0 & 100 \\
\hline Stephanolepis diaspros* & Monacanthidae & Disc. & 450 & - & 0 & 100 \\
\hline Bothus podas & Bothidae & Disc. & 427 & - & 0 & 100 \\
\hline Spicara maena & Centracanthidae & Minor & 406 & 130 & 42 & 89.6 \\
\hline Trachurus picturatus & Carangidae & Com. & 342 & 130 & 186 & 45.6 \\
\hline Trachurus trachurus & Carangidae & Com. & 332 & 130 & 240 & 27.7 \\
\hline Capros aper & Caproidae & Disc. & 325 & - & 0 & 100 \\
\hline Sardinella aurita & Clupeidae & Com. & 319 & $110^{* *}$ & 217 & 32.0 \\
\hline Sphyraena sphyraena & Sphyraenidae & Com. & 286 & 150 & 268 & 6.3 \\
\hline Arnoglossus sp. & Bothidae & Disc. & 213 & - & 0 & 100 \\
\hline Uranoscopus scaber & Uranoscopidae & Disc. & 203 & - & 0 & 100 \\
\hline Scomber japonicus & Scombridae & Com. & 197 & 110 & 172 & 12.7 \\
\hline Pagrus coeruleostictus & Sparidae & Com. & 186 & 110 & 116 & 37.6 \\
\hline Diplodus sargus & Sparidae & Com. & 165 & $110^{* *}$ & 144 & 12.7 \\
\hline Synodus saurus & Synodontidae & Com. & 159 & $160 * *$ & 108 & 32.0 \\
\hline Ariosoma balearicum & Congridae & Disc. & 152 & - & 0 & 100 \\
\hline Trigloporus lastoviza & Triglidae & Disc. & 149 & - & 0 & 100 \\
\hline Diplodus annularis & Sparidae & Com. & 138 & $110^{* *}$ & 87 & 36.9 \\
\hline Trigla lyra & Triglidae & Disc. & 130 & - & 0 & 100 \\
\hline Caranx rhonchus & Carangidae & Minor & 123 & 130 & 110 & 10.5 \\
\hline Serranus cabrilla & Serranidae & Disc. & 113 & - & 0 & 100 \\
\hline Gobius niger & Gobiidae & Disc. & 110 & - & 0 & 100 \\
\hline Sillago sihama* & Sillaginidae & Com. & 90 & 130 & 73 & 18.8 \\
\hline Spicara flexиоsa & Centracanthidae & Minor & 86 & 130 & 16 & 81.4 \\
\hline Helicolenus dactylopterus & Scorpaenidae & Disc. & 85 & - & 0 & 100 \\
\hline Epinephelus aeneus & Serranidae & Com. & 79 & 150 & 53 & 32.9 \\
\hline Lagocephalus suezensis* & Tetraodontidae & Disc. & 78 & - & 0 & 100 \\
\hline Seriola dumerili & Carangidae & Com. & 76 & 130 & 70 & 7.9 \\
\hline Scomberomorus commerson* & Scombridae & Com. & 73 & 140 & 67 & 8.2 \\
\hline Lepidorhombus whiffiagonis & Scophthalmidae & Minor & 71 & 150 & 0 & 100 \\
\hline Trichiurus lepturus & Trichiuridae & Minor & 64 & 300 & 47 & 26.6 \\
\hline Solea spp. & Soleidae & Minor & 60 & 150 & 24 & 0.6 \\
\hline Diplodus vulgaris & Sparidae & Com. & 57 & $110 * *$ & 48 & 15.8 \\
\hline Chlorophthalmus agassizii & Chlorophthalmidae & Disc. & 56 & - & 0 & 100 \\
\hline Pomadasys incisus & Haemulidea & Minor & 55 & 130 & 45 & 18.2 \\
\hline Atherina spp. & Atherinidae & Disc. & 49 & - & 0 & 100 \\
\hline Trachinus araneus & Trachinidae & Disc. & 48 & - & 0 & 100 \\
\hline Apogon imberbis & Apogonidae & Disc. & 44 & - & 0 & 100 \\
\hline Gnatholepis mystax & Congridae & Disc. & 38 & - & 0 & 100 \\
\hline Phycis phycis & Gadidae & Disc. & 37 & - & 0 & 100 \\
\hline Dussumieria elopsoides* & Clupeidae & Minor & 36 & $110 * *$ & 36 & 0 \\
\hline Herklotsichthys punctatus* & Clupeidae & Minor & 34 & 110 & 0 & 100 \\
\hline Pagrus pagrus & Sparidae & Minor & 33 & 110 & 1 & 96.9 \\
\hline Coelorhynchus Coelorhynchus & Macrouridae & Disc. & 25 & - & 0 & 100 \\
\hline Zeus faber & Zeidae & Minor & 25 & 120 & 4 & 84.0 \\
\hline Oxyuirichthys petersi* & Gobiidae & Disc. & 24 & - & 0 & 100 \\
\hline Caranx crysos & Carangidae & Com. & 21 & 130 & 15 & 28.6 \\
\hline Lagocephalus spadiceus* & Tetraodontidae & Disc. & 21 & - & 0 & 100 \\
\hline Siganus rivulatus* & Siganidae & Minor & 20 & 130 & 5 & 0.75 \\
\hline Sargocentron rubrum* & Holocentridae & Minor & 18 & 140 & 17 & 5.5 \\
\hline
\end{tabular}


TABLE 1 (cont.). - List of species recorded in surveys, their origin, commercial value and abundance in 324 trawl samples, as well as their landed and discarded fractions in the catch. N, number of specimens; Minor, minor commercial value; Com., commercial; Disc., discarded; * Lessepsian migrant; ** discard size was set according to MLS regulations; *** species were not separated into samples and were recorded as one taxa.

\begin{tabular}{|c|c|c|c|c|c|c|}
\hline Species & Family & Value & $\mathrm{N}$ & $\begin{array}{c}\text { Discard } \\
\text { Size in } \mathrm{mm}\end{array}$ & $\begin{array}{l}\text { No. } \\
\text { Landed }\end{array}$ & Discard \% \\
\hline Diplodus puntazzo & Sparidae & Com. & 17 & $110 * *$ & 17 & 0 \\
\hline Ophiodon barbatum & Ophidiidae & Disc. & 17 & - & 0 & 100 \\
\hline Balistes capriscus & Balistidae & Com. & 16 & 130 & 14 & 12.5 \\
\hline Microchirus ocellatus & Soleidae & Disc. & 14 & - & 0 & 100 \\
\hline Dactylopterus volitans & Dactylopteridae & Minor & 13 & 130 & 2 & 84.6 \\
\hline Oblada melanura & Sparidae & Com. & 13 & $110 * *$ & 12 & 7.7 \\
\hline Cynoglossus sinusarabici* ${ }^{*}$ & Cynoglossidae & Disc. & 11 & - & 0 & 100 \\
\hline Terapon puta* & Terapontidae & Disc. & 10 & - & 0 & 100 \\
\hline Liza aurata & Mugilidae & Com. & 9 & $200 * *$ & 5 & 4.44 \\
\hline Dentex gibbosus & Sparidae & Minor & 8 & 110 & 8 & 0 \\
\hline Aspitrigla cuculus & Triglidae & Disc. & 7 & - & 0 & 100 \\
\hline Conger conger & Congridae & Disc. & 7 & - & 0 & 100 \\
\hline Dentex dentex & Sparidae & Minor & 7 & 110 & 2 & 71.4 \\
\hline Dentex maroccanus & Sparidae & Minor & 7 & 110 & 1 & 85.7 \\
\hline Hoplostethus mediterraneus & Trachichthyidae & Disc. & 6 & - & 0 & 100 \\
\hline Sparus aurata & Sparidae & Com. & 6 & 110 & 6 & 0 \\
\hline Xyrichthys novacula & Labridae & Disc. & 6 & - & 0 & 100 \\
\hline Blennius ocellaris & Blenniidae & Disc. & 5 & - & 0 & 100 \\
\hline Epinephelus haifensis & Serranidae & Minor & 5 & 150 & 4 & 0.2 \\
\hline Alectis alexandrines & Carangidae & Minor & 4 & 150 & 0 & 100 \\
\hline Apogon pharonis* & Apogonidae & Disc. & 4 & - & 0 & 100 \\
\hline Pempheris vanicolensis* & Pempheridae & Disc. & 4 & - & 0 & 100 \\
\hline Siganus luridus* & Siganidae & Minor & 4 & 130 & 0 & 100 \\
\hline Trachinus radiatus & Trachinidae & Disc. & 4 & - & 0 & 100 \\
\hline Trigla lucerna & Triglidae & Disc. & 4 & - & 0 & 100 \\
\hline Dicentrarchus punctatus & Moronidae & Minor & 3 & 150 & 3 & 0 \\
\hline Hyporamphus affinis* & Hemiramphidae & Minor & 3 & 160 & 3 & 0 \\
\hline Lepidopus caudatus & Trichiuridae & Minor & 3 & 300 & 3 & 100 \\
\hline Liza ramada & Mugilidae & Minor & 3 & 200 & 2 & 33.3 \\
\hline Lophius budegassa & Lophiidae & Minor & 3 & 200 & 3 & 100 \\
\hline Torquigener flavimaculosus* & Tetraodontidae & Disc. & 3 & - & 0 & 100 \\
\hline Anguilla Anguilla & Anguillidae & Disc. & 2 & - & 0 & 100 \\
\hline Argyrosomus regius & Sciaenidae & Com. & 2 & 150 & 2 & 0 \\
\hline Etrumeus teres* & Clupeidae & Com. & 2 & 150 & 2 & 0 \\
\hline Hemiramphus far* & Hemiramphidae & Minor & 2 & 160 & 2 & 0 \\
\hline Muraena Helena & Muraenidae & Minor & 2 & - & 0 & 100 \\
\hline Phycis blennoides & Gadidae & Disc. & 2 & - & 0 & 100 \\
\hline Pomatomus saltator & Pomatomidae & Com. & 2 & 150 & 2 & 0 \\
\hline Argentina sphyraena & Argentinidae & Minor & 1 & - & 0 & 100 \\
\hline Echelus myrus & Ophichthidae & Disc. & 1 & - & 0 & 100 \\
\hline Echeneis naucrates & Echeneididae & Disc. & 1 & - & 0 & 1 \\
\hline Epinephelus costae & Serranidae & Minor & 1 & 150 & 1 & 0 \\
\hline Lepidotrigla dieuzeidei & Triglidae & Disc. & 1 & - & 0 & 100 \\
\hline Microchirus variegates & Soleidae & Disc. & 1 & - & 0 & 100 \\
\hline Mugil cephalus & Mugilidae & Com. & 1 & 200 & 1 & 0 \\
\hline Pagrus auriga & Sparidae & Com. & 1 & 110 & 1 & 0 \\
\hline Panturichthys fowleri & Heterechelyidae & Disc. & 1 & - & 0 & 100 \\
\hline Scorpaena notate & Scorpaenidae & Disc. & 1 & - & 0 & 100 \\
\hline Scorpaena scrofa & Scorpaenidae & Disc. & 1 & - & 0 & 100 \\
\hline Symphurus nigrescens & Cynoglossidae & Disc. & 1 & - & 0 & 100 \\
\hline \multicolumn{7}{|l|}{ Cartilagenous Fishes } \\
\hline Torpedo torpedo & Torpedinidae & Minor & 30 & 170 & 14 & 53.3 \\
\hline Rhinobatos rhinobatos & Rhinobatidae & Com. & 8 & 300 & 3 & 62.5 \\
\hline Raja miraletus & Rajidae & Com. & 7 & 250 & 0 & 100 \\
\hline Squalus blainvillei & Squalidae & Minor & 3 & 500 & 2 & 33.3 \\
\hline Torpedo marmorata & Torpedinidae & Minor & 3 & 170 & 2 & 33.3 \\
\hline Dasyatis pastinaca & Dasyatidae & Com. & 2 & 250 & 1 & 50 \\
\hline Mustelus mustelus & Triakidae & Minor & 1 & 500 & 0 & 100 \\
\hline \multicolumn{7}{|l|}{ Cephalopods } \\
\hline Loligo vulgaris & Loliginidae & Com. & 1481 & 100 & 233 & 84.3 \\
\hline Sepia officinalis & Sepiidae & Com. & 242 & 60 & 189 & 21.9 \\
\hline Octopus/Eledone sp. & Octopodidae & Com. & 134 & 20 & 100 & 25.4 \\
\hline Illex coindeti & Ommastrephidae & Com. & 63 & 100 & 20 & 68.2 \\
\hline \multicolumn{7}{|l|}{ Crustaceans } \\
\hline Parapenaeus longirostris & Penaeidae & Com. & 14773 & 60 & 4253 & 71.5 \\
\hline Marsupenaeus japonicus* & Penaeidae & Com. & 3707 & 60 & 3637 & 1.9 \\
\hline Charybdis longicollis* & Portunidae & Disc. & 3595 & - & 0 & 100 \\
\hline Aristeomorpha foliaceal & & & & & & \\
\hline Aristeus antennatus $* * *$ & Aristeidae & Com. & 2940 & 60 & 2270 & 22.8 \\
\hline Erugosquilla massavensis*/ & & & & & & \\
\hline Squilla mantis*** & Squillidae & Disc. & 252 & - & 0 & 100 \\
\hline Portunus pelagicus* & Portunidae & Com. & 30 & 70 & 30 & 0 \\
\hline Penaeus semisulcatus* & Penaeidae & Com. & 41 & 60 & 41 & 0 \\
\hline Pontocaris cataphracta & Crangonidae & Disc. & 12 & - & 0 & 100 \\
\hline Metapenaeus monoceros* & Penaeidae & Minor & 8 & 60 & 8 & 0 \\
\hline
\end{tabular}

SCI. MAR., 75(4), December 2011, 641-652. ISSN 0214-8358 doi: 10.3989/scimar.2011.75n4641 
b. Biomass by Depth - 1990-4

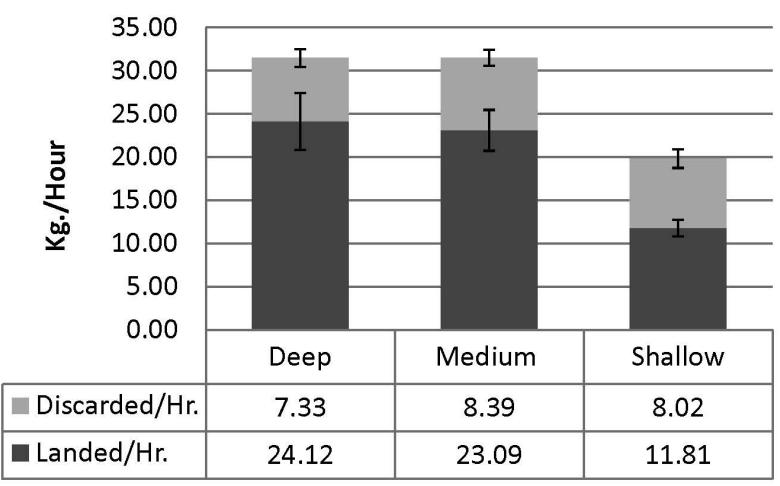

d. Biomass by Area - 1990-4

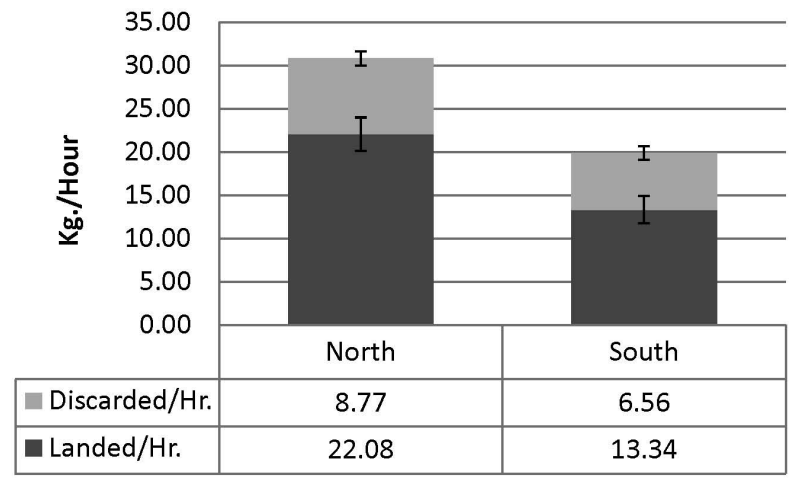

a. Biomass by Season - 1990-4

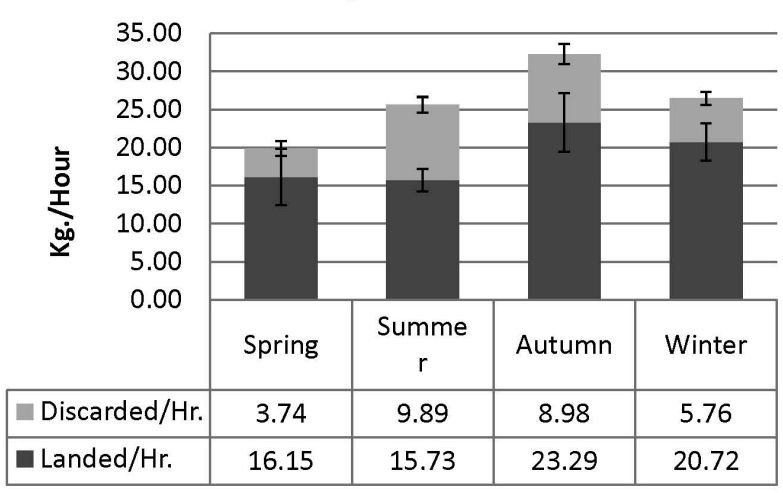

c. Biomass by Daytime - 1990-4

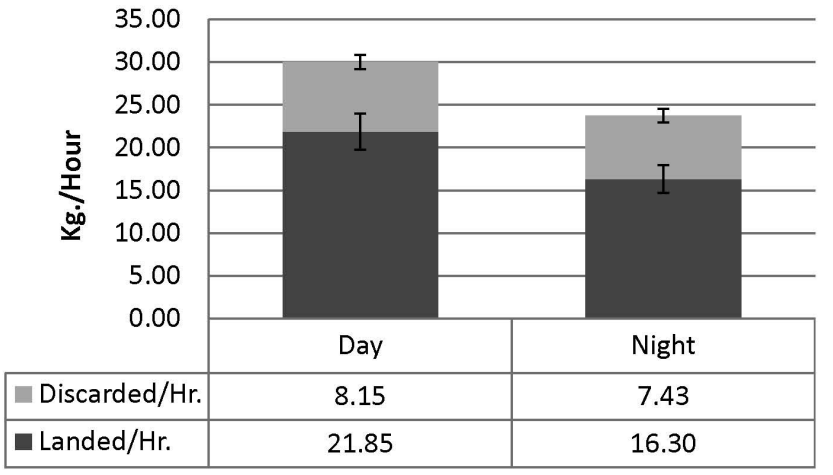

FIG. 3. - Mean landed and discarded biomass \pm standard error, recorded in 173 trawl hauls for the 4 study factors: a, season; b, depth; c, time of day; and d, area.

TABLE 2. - Number of hauls used for the analysis of sample data (total $\mathrm{n}=324$ ) and biomass (recorded in 173 hauls). Note that several hauls may have exceeded a single time of day, depth or area and were thus omitted from the respective analysis.

\begin{tabular}{lccc}
\hline $\begin{array}{l}\text { Sample analysis } \\
(\text { Total } \mathrm{n=324)}\end{array}$ & $\begin{array}{c}\text { Biomass analysis } \\
\text { (Total } \mathrm{n}=173)\end{array}$ & Details & Factor \\
\hline 78 & 40 & Oct.-Dec. & Autumn \\
100 & 53 & Jan.-Mar. & Winter \\
40 & 17 & Apr.-Jun. & Spring \\
106 & 63 & Jul.-Sep. & Summer \\
55 & 38 & $>84 \mathrm{~m}$ & Deep \\
147 & 72 & $38-83 \mathrm{~m}$ & Medium \\
114 & 57 & $<37 \mathrm{~m}$ & Shallow \\
162 & 90 & Daylight & Day \\
158 & 82 & Darkness & Night \\
199 & 106 & North of Hadera & North \\
116 & 60 & South of Hadera & South \\
\hline
\end{tabular}

$H^{\prime}=1.96$ and $S=18.17$ species per haul in the summer to values of 1.77 and 15.72 respectively in winter.

The discard ratio was also found to be highest in shallow fishing fields (Fig. 3b; 40.1\%) and discards were comprised mostly of unwanted species (Fig. 4b). In deeper strata, discard proportions were lower but were comprised mostly of juveniles of commercial species. Diversity and richness were negatively correlated with depth, and decreased from 1.92 and 17.94 for shallow hauls (<37 $\mathrm{m}$ deep) to 1.56 and $14.98 \mathrm{spe}-$ cies per haul in deep hauls respectively.
TABLE 3. - A permutation analysis of variance (PERMANOVA) designed to test interactions of the study factors - depth, time of day (nested in depth), area and season, and their interactions. $\mathrm{P}($ perm) indicates the statistical significance of the interaction. $* \mathrm{P}<0.05$ $* * \mathrm{P}<0.005$

\begin{tabular}{lcclc}
\hline Factor & df & Pseudo-F & P(perm) & perms \\
\hline Depth & 2 & 4.1055 & $0.018 *$ & 999 \\
Area & 2 & 1.5092 & 0.145 & 998 \\
Season & 3 & 2.8254 & 0.102 & 997 \\
Time of day (Depth) & 3 & 4.3359 & $0.001 * *$ & 999 \\
Depth×Area & 3 & 1.4737 & 0.134 & 999 \\
Depth×Season & 6 & 2.0571 & 0.079 & 999 \\
Area×Season & 5 & 1.5973 & 0.219 & 998 \\
Area×Time of day(Depth) & 4 & 1.5736 & $0.008 *$ & 999 \\
Season×Time of day(Depth) & 7 & 1.7061 & $0.001 * *$ & 996 \\
Depth×Area×Season & 5 & 1.3105 & 0.302 & 999 \\
Area×Season×Time of day (Depth) & 3 & 1.4126 & $0.04 *$ & 997 \\
Residual & 274 & $4.1967 \mathrm{E} 5$ & & \\
Total & 324 & $8.1654 \mathrm{E} 5$ & & \\
\hline
\end{tabular}

The predominance of night hauls in shallow waters (80 out of 114 shallow hauls were nocturnal; Fig. 5) caused the depth and time of day to be confounding. The time of day factor was therefore nested within the depth factor in the permutational analysis of variance (PERMANOVA) design, which tested for interactions between factors. The PERMANOVA model results (Table 3) indicate that depth and time of day nested within depth had the most significant effects on catch 
a. Discarded Specimens (fish only) by Season

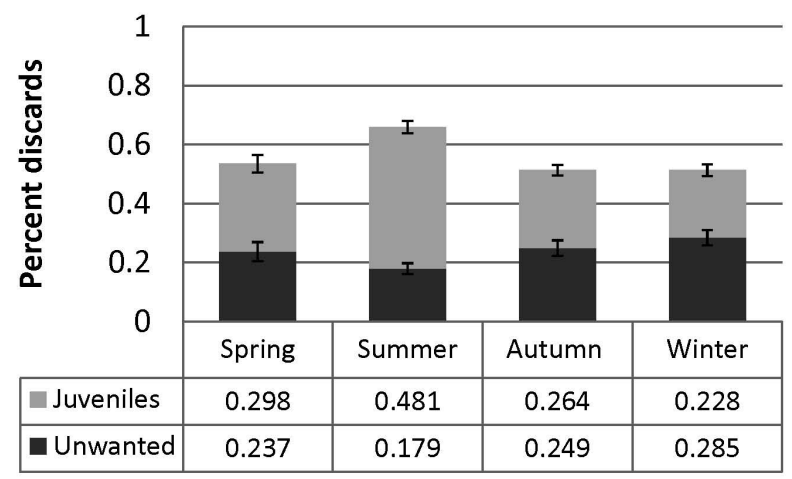

c. Discarded Specimens (fish only) by Daytime

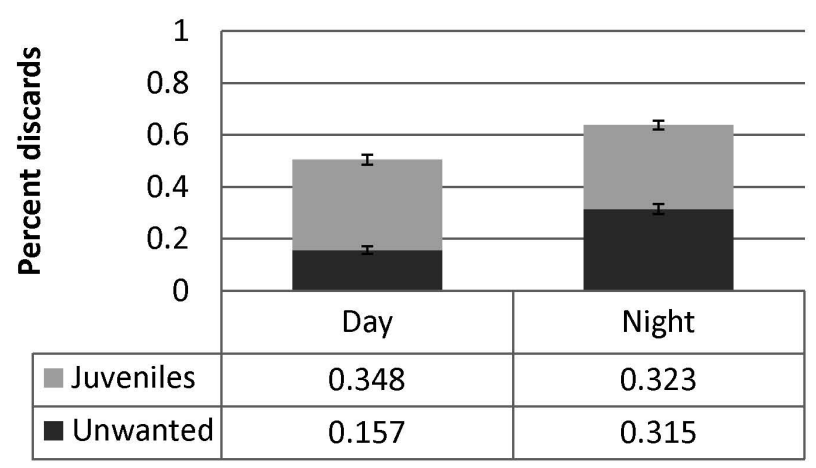

\section{b. Discarded Specimens (fish only) by Depth}

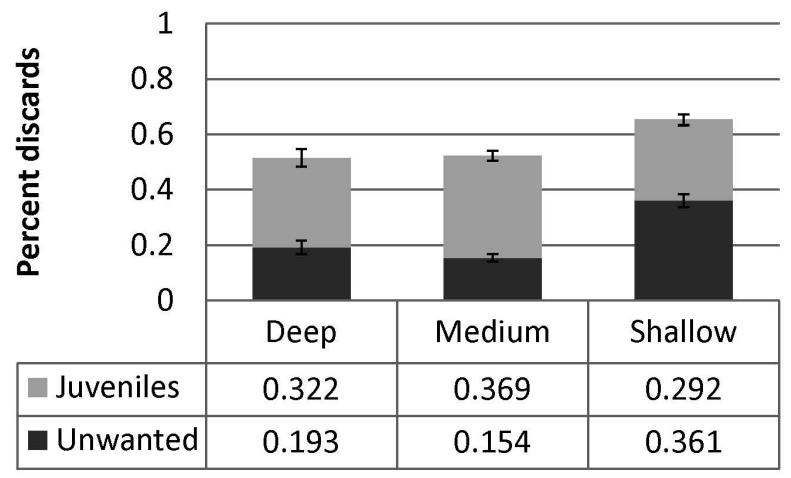

d. Discarded Specimens (fish only) by Area

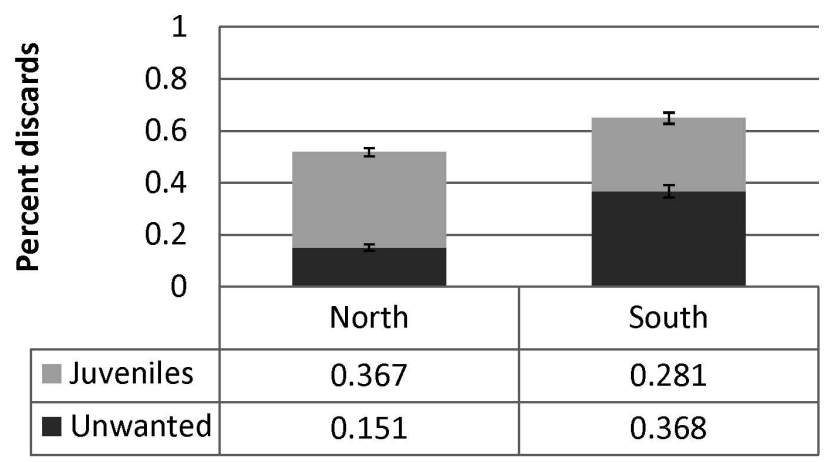

FIG. 4. - Mean proportion of the two fractions comprised in the discarded portion of the fish in 324 haul samples: undersized juveniles of commercial species and specimens of unwanted, non-commercial species \pm standard error, for the 4 study factors: a, season; b, depth; c, time of day; and d, area.

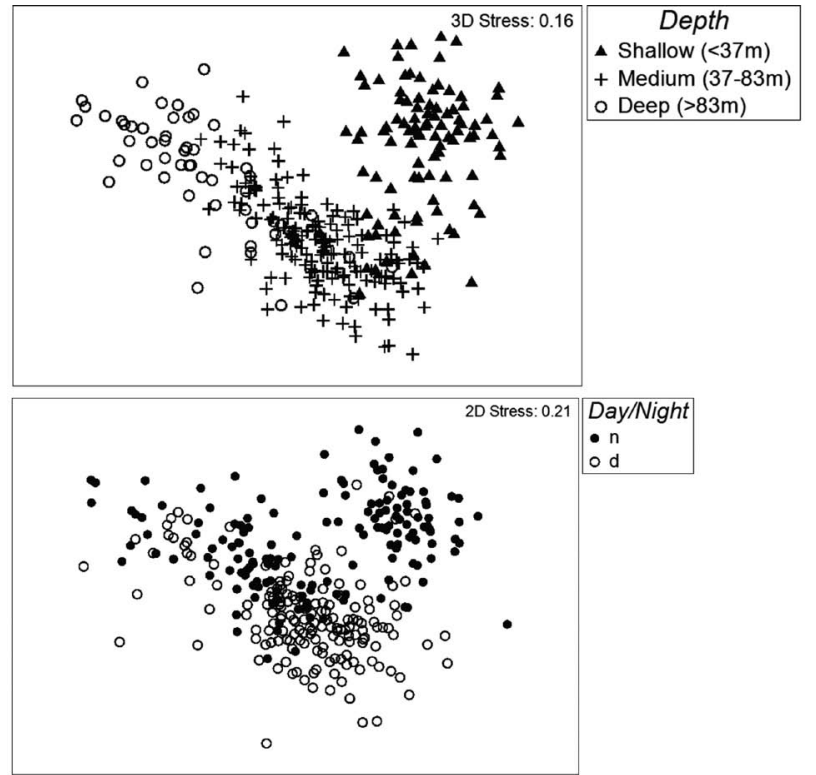

FIG. 5. - MDS of the species composition of 324 haul samples according to haul depth (top) and time of day (bottom); n, night; d, day.

species composition. Towing area and season had no significant effects on composition, except when they interacted with the time of day nested in depth, which suggests a complex set of influences on species compositions. After it was determined that depth was the dominant factor affecting catch composition, a SIMPER analysis was performed separately for each of the 3 depth strata. Higher similarity (Table 4) was recorded for hauls in deep and medium strata than in shallow hauls. The porgy Pagellus erythrinus was found to be the most dominant species in shallow and medium depths. Commercial penaeid prawns were among the dominant species in both shallow and deep hauls, with $P$. longirostris and $M$. japonicus ranking first and third respectively in these strata.

Several trends were found to differentiate between trawling areas: northern hauls showed higher landed catches (by $9 \mathrm{~kg} \mathrm{~h}^{-1}$; Fig. 3d) and lower biomass discard percentages (by 6\%) than southern hauls. However, these discards were comprised mainly of juveniles of commercial species (Fig. 4d).

Migrants accounted for $29.2 \%$ of the total landed biomass in surveys. Out of 26 Lessepsian bony fish species, 7 were commercial, 6 were of minor commercial value and 13 were always discarded (Table 1), whereas 4 out of the 6 Lessepsian crustacean species were of commercial value. In The SIMPER analysis, 4 out of the 7 most dominant species in the shallow stratum were Lessepsian migrants (Table 4). In depths exceeding $37 \mathrm{~m}$, the dominant Lessepsian species were 
TABLE 4. - Results of a nonparametric SIMPER procedure based on a square root transformed Bray-Curtis similarity index of the number of specimens per hour of trawling. The contribution of the dominant species to the similarity \% (Contrib\%) of the 3 depth strata is described for the 7 dominant species of each stratum. Other parameters include the average contribution to abundance, similarity, similarity/standard deviation and cumulative similarity.

\begin{tabular}{|c|c|c|c|c|c|}
\hline Species & Av.Abund & Av.Sim & $\mathrm{Sim} / \mathrm{SD}$ & Contrib\% & Cum. $\%$ \\
\hline \multicolumn{6}{|c|}{ Shallow stratum $(15-37 \mathrm{~m})$ average similarity: 30.31} \\
\hline Pagellus erythrinus & 1.50 & 6.30 & 1.25 & 20.77 & 20.77 \\
\hline Callionymus filamentosus* & 1.30 & 5.41 & 0.97 & 17.84 & 38.61 \\
\hline Marsupenaeus japonicus* & 1.26 & 4.86 & 0.70 & 16.05 & 54.66 \\
\hline Boops boops & 0.68 & 2.17 & 0.78 & 7.15 & 61.81 \\
\hline Saurida undosquamis* & 0.52 & 1.33 & 0.66 & 4.39 & 66.19 \\
\hline Lithognathus mormyrus & 0.50 & 1.32 & 0.67 & 4.36 & 70.55 \\
\hline Upeneus pori* & 0.45 & 1.26 & 0.69 & 4.17 & 74.72 \\
\hline \multicolumn{6}{|c|}{ Medium stratum (38-83 m) average similarity: 34.06} \\
\hline Pagellus erythrinus & 1.46 & 6.01 & 1.72 & 17.65 & 17.65 \\
\hline Upeneus moluccensis* & 1.51 & 5.21 & 0.98 & 15.28 & 32.93 \\
\hline Mullus barbatus & 1.20 & 3.25 & 0.79 & 9.54 & 42.48 \\
\hline Boops boops & 1.16 & 3.18 & 0.86 & 9.33 & 51.81 \\
\hline Mullus surmuletus & 0.99 & 2.27 & 0.64 & 6.68 & 58.49 \\
\hline Pagellus acarne & 0.88 & 1.60 & 0.50 & 4.70 & 63.19 \\
\hline Saurida undosquamis* & 0.62 & 1.51 & 0.59 & 4.43 & 67.62 \\
\hline \multicolumn{6}{|c|}{ Deep stratum (>84 m) average similarity: 33.09} \\
\hline Parapenaeus longirostris & 2.77 & 12.63 & 1.13 & 38.18 & 38.18 \\
\hline Merluccius merluccius & 1.09 & 4.33 & 1.01 & 13.07 & 51.25 \\
\hline Dentex macrophthalamus & 1.23 & 3.54 & 0.66 & 10.69 & 61.93 \\
\hline Boops boops & 0.85 & 1.94 & 0.60 & 5.87 & 67.80 \\
\hline Mullus barbatus & 0.62 & 1.77 & 0.75 & 5.35 & 73.15 \\
\hline Macrohamphosus scolopax & 0.77 & 1.48 & 0.53 & 4.47 & 77.62 \\
\hline Aristeomorpha foliacea & 0.77 & 1.24 & 0.28 & 3.73 & 81.35 \\
\hline
\end{tabular}

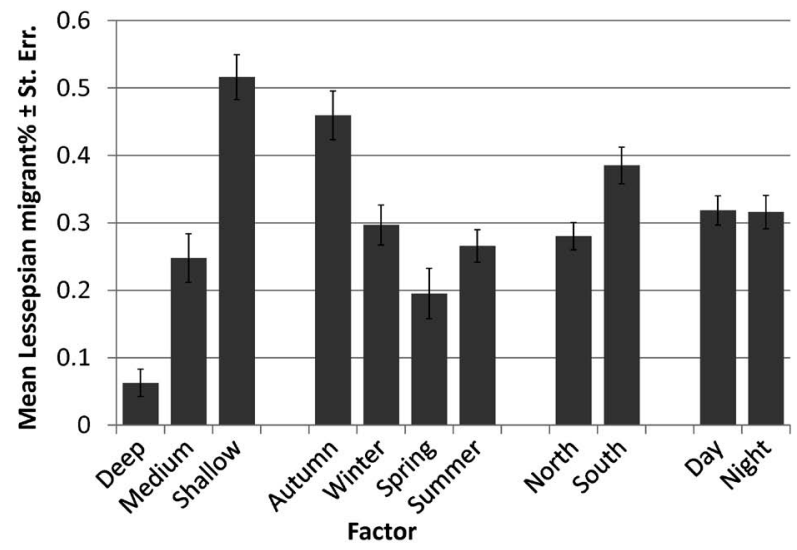

FIG. 6. - Mean Lessepsian migrant proportion \pm S.E. for the 4 study factors in the 324 haul samples.

the goatfish U. moluccensis and the lizardfish S. undosquamis (Table 4). In shallower waters, where migrants proliferate, the penaeid prawn M. japonicus was the prominent commercial species. It also accounted for the bulk of shallow landings. Other common migrants were mostly non-commercial species such as the inshore pelagic pony fish L.klunzingeri, the shallow water dragonet $C$. filamentosus and the swimming crab $C$. longicollis. Proportions of migrants in the catch varied significantly (Wilcoxon, $\mathrm{P}<0.05$ ) with season, depth and area, peaking in the autumn, shallows and south respectively (Fig. 6). No statistically significant differences were found for the time of day. In total, $43.9 \%$ of all Indo-Pacific specimens were discarded; slightly less than the $47.3 \%$ noted for indigenous specimens.

\section{DISCUSSION}

\section{Discards and catch composition}

This is the first fully quantitative study of demersal fish communities in the Levant. Despite its delayed publication, this dataset provides a crucial reference point for the assessment of change vectors. It also demonstrates the magnitude of the effects of trawling on the Levantine ecosystem via discards, which is a prime conservation issue. Discards in the present study amounted to $28.2 \%$ of the total catch, or 330-550 t of the 1107$1757 \mathrm{t} \mathrm{y}^{-1}$ caught by Israeli trawlers during the study period (Snovsky and Shapiro, 1997). It is estimated, however, that $>90 \%$ of trawling in Israel is performed at depths shallower than $83 \mathrm{~m}$, where discard rates are closer to $40 \%$ (Fig. 3b). This leads to a total discards estimate of 440-700 t per annum for the Israeli fleet. The $28.2 \%$ figure for total discards in the present study is considerably lower than the $40-45 \%$ figure used by Davies et al. (2009) for the entire Mediterranean, but higher than the $15-25 \%$ values suggested by El-Mor et al. (2002) and Alsayes et al. (2009) for Egyptian trawlers. The high discard ratios described here clearly support the use of more selective trawl nets, such as the $40 \mathrm{~mm}$ square mesh nets mandated by the General Fisheries Commission for the Mediterranean (GFCM) for Mediterranean fleets by 2012. Mesh size regulations in Israel today are still similar to those used during the study period. We therefore recommend making further changes, as well as establishing MPAs and a summer moratorium on trawling as precautionary measures. 
Israeli gross catch rates fluctuated seasonally between 19.9 and $32.2 \mathrm{~kg} \mathrm{~h}^{-1}$, while discards varied between 3.7 and $9.9 \mathrm{~kg} \mathrm{~h}^{-1}$ (Fig. 3). Despite the poor Levantine fishery suppositions made in various studies (Por, 1989, Sonin et al., 2007), these values are comparable to catch and discard rates reported for the central Mediterranean (Machias et al., 2001, D'Onghia et al., 2003, Sánchez et al., 2007). Discarded biomass alone, however, cannot represent the full extent of the impact of trawling on future year classes. The spatiotemporal variance in discards, as shown in Fig. 4, reveals the motive for discarding and also the potential commercial losses incurred. We suggest that these datasets may compensate slightly for the absence of proper stock assessments in the region. Nearly $60 \%$ of all fish were discarded in the present study, with juveniles of commercially exploited species representing $>60 \%$ of these discards.

97 species appeared in both Machias et al. (2001) and the present study. Out of these, 30 taxa were marketed in Greece but discarded in Israel. An example of these taxa (which abound in catches but are seldom sold) is the genus Spicara ( $\mathrm{n}=2978$ in Table 1). Members of this genus comprise up to $50 \%$ of the commercial catch of trawls in Cyprus (Garcia and Demetropoulos, 1986), while they are normally discarded in every size in Israel, and only acquire a minor commercial value when catches are especially poor. Other such species include congers, ophiodons and other eels, gurnards, some flatfishes, such as B. podas or $C$. linguatula, scorpion fishes, weavers and stargazers. Some crustaceans with commercial potential are also discarded in Israel, most notably the stomatopod Squilla mantis, which is landed consistently, and seldom discarded in Balearic and Adriatic trawl fisheries (Sánchez et al., 2007, Demestre et al., 2008). The Israeli fishing fleet is thus wasting a potentially valuable resource, as these species can be landed and sold if a suitable market can be found. However, when this issue is addressed the smaller size of Levantine organisms (i.e. Nanism), may limit the commercial potential of these genera. This dwarfing phenomenon has been suggested to be the result of lower productivity in the region (Sonin et al., 2007) and is speculated to affect most of the Levantine fauna (Por, 1989, Sonin et al., 2007).

For several species, most noticeably goatfishes, the observed discard length differed greatly from MLS. The MLS of goatfish is $11 \mathrm{~cm}$; however, due to the high prices obtained for goatfish regardless of size, specimens as small as $9 \mathrm{~cm}$ were consistently landed and this was the length actually used as the maximum discard size. This led to fairly low discard rates of $17.5-27.3 \%$ for the 4 mullid species. An examination of the most commonly caught commercial and minorcommercial species in Table 1 reveals two distinct subgroups. One subgroup includes species which exhibit low discard rates (less than 30\%) such as the 4 mullid species, lizardfish and hake (due to their high commercial value and small discard sizes), rock-dwelling sea breams (most of which are adults caught foraging slightly off their rocky habitat) and pelagic or semipelagic families such as Scombridae, Clupeidae or Carangidae. The second subgroup is made up of species for which $60-98 \%$ of the specimens were discarded. This includes some of the most common species, such as porgies, bouge, picarels, dentex and flatfishes. The landing size for these species is substantially larger than the mean size of caught specimens, which leads to high discard rates. Esmeralda Costa et al. (2009) found a similar trend as far west as Portugal, with less than $30 \%$ discards for sardines, carangids, scombrids and Diplodus spp., as opposed to $>70 \%$ for porgies and hake. It is evident from the small size of the fish caught by Levantine trawlers (Table 1) that most individuals have not reached reproductive maturity. This seriously jeopardizes the sustainability of the fishery regardless of whether juveniles are landed or discarded.

\section{Spatial and temporal trends}

Depth was found to be the main explanatory factor for both catch composition and discard percentage. Higher landed and lower discarded biomass was found deeper than $37 \mathrm{~m}$ (Fig. 3b). However, the proportion of juveniles of commercial species within discards in these deep strata was significantly higher (Fig. 4). Shallow stratum discard proportions were higher (Fig. $3 \mathrm{~b}$ ); however, they were dominated by non-commercial species, rather than commercial juveniles (Fig. 4b). This finding may present a dilemma for fishery managers seeking to minimize discards via spatial exclusion: although closing shallow waters to trawling will lead to a richer and more diverse environment, shifting the effort into deeper grounds will harm more commercial juveniles. A trawl ban shallower than $50 \mathrm{~m}$ (as described by Sweeting et al., 2009) may not be suitable for Israeli fishing fields, as it may displace trawling effort into deeper grounds, where juvenile recruits make up a larger portion of the assemblage. In addition, a $50 \mathrm{~m}$ minimum trawl depth would eliminate the entire $M$. japonicus catch, the most valuable commercial resource harvested by trawlers.

High summer discard proportions were accompanied by increases in Shannon's H' and species richness. As in shallow depths, summer discards were comprised mostly of non-commercial species (Fig. 4a). A summer trawling moratorium, as practiced in other Mediterranean countries (Machias et al., 2001, D'Onghia et al., 2003, Sánchez et al., 2004, Demestre et al., 2008) may prove effective in raising CPUE and reducing discards. An effective moratorium requires that fishing pressure is not merely shifted to other seasons and that discard practices remain consistent throughout the fishing season (Machias et al., 2001, Sánchez et al., 2007).

As in the present study, Sánchez et al. (2004) reported that discarded biomass decreased with depth on the shelf. Machias et al. (2001) and D'Onoghia et al. (2003), working on the slope, found that discard 
percentages increased with depth. This may attest to a spatial discarding pattern for the Mediterranean, where discards are highest on the deep shelf and decrease towards the shallower shelf from one end and the deeper slope on the other.

Juveniles of the European squid L. vulgaris and the rose shrimp $P$. longirostris were dominant among deep strata discards. Whereas squids are consistently landed when of size, rose shrimps are often discarded even at legal sizes, mostly due to low market prices. Despite the high abundance of rose shrimp, the high costs of deep sea trawling often stop trawlers from targeting it. Conversely, discarded crustaceans in shallow waters included mostly the non-commercial swimming crab C. longicollis. Along with several other species, this crab was responsible for a high crustacean discard rate in Levantine shallow waters $\left(>5 \mathrm{~kg} \mathrm{~h}^{-1}\right)$, which is unlike the low ca. $1 \mathrm{~kg} \mathrm{~h}^{-1}$ crustacean discard rate noted by Machias et al. (2001) in Greece.

\section{Lessepsian migrants}

The percent of Lessepsian migrant specimens in trawl catches decreased from $51 \%$ in the shallows to $24 \%$ and $8 \%$ in the medium and deep strata respectively. S. undosquamis and $U$. moluccensis were the dominant Indo-Pacific species in the deeper grounds; perhaps outlining the maximum bathymetric boundary of migration. The niche partitioning pattern, (Golani, 1993) which suggests that native goatfishes dominate deeper strata than migrants, is also evident in the present data (Table 4).

While Lessepsian migrants remain poorly established in the rocky littoral (Golani et al., 2007), this study has found that they proliferate in highly (and often) disturbed trawl grounds. This heavily exploited environment only allows resilient species with rapid population recovery rates and recolonization abilities to persist. We therefore hypothesize that Lessepsian migrants display good adaptive characteristics, which have enabled them to migrate and colonize a new ecosystem, and which have given them a selective advantage in a scheme of recurring and rapid exploitation and recolonization like that imposed by trawling.

Lessepsian migrants were a significant and valuable portion of the Israeli trawl catch in the past (BenYami, 1955), and remain so today (Sonin et al., 2007). Still, some studies argue that the cumulative effect of migration is harmful (Galil, 2007). Ben-Tuvia (1973) estimated that migrants constitute $21 \%$ of trawl yields, slightly less than the landed migrant biomass proportion found in the present study (29\%). As expected, migrants were most common in shallow grounds (Fig. 6). No substantial diurnal differences in migrant proportions were observed; however, their composition in catches varied between night and day. The SIMPER analysis showed that typical nighttime migrants were the dragonet $C$. filamentosus and the prawn $M$. japonicus in the shallows. The main migrants contributing to daytime assemblages in all strata were $U$. moluccensis, $S$. undosquamis and L. klunzingeri. The first two species were also responsible for high migrant percentages in autumn and winter (Fig. 6). These species, along with the indigenous $B$. boops and P. erythrinus, also accounted for the high landings in autumn (Fig. 3a). The present study has also found a significantly higher proportion of Lessepsian migrants in the southern trawling area (Fig. 6). We suggest that this is either the result of its proximity to the Suez Canal or of the wider continental shelf of southern Israel.

Today, the migrant prawn M. japonicus is a central target species of the Egyptian, Turkish and Israeli trawl fisheries (Galil, 2007). Its high market value and nearshore habitat have reshaped local trawling and turned the shallow strata into the most intensively trawled habitat off the Israeli coast. Since its acceptance in the local market in the 1970s, it has comprised more than a fifth of the income of Israeli trawlers (Snovsky and Shapiro, 1997). With increasing migration rates, soaring oil prices and declines in deep fisheries, it stands to reason that the market value and overall biomass of migrant species will continue to rise in the future. More research and time are required to determine whether migrants can compensate for the loss of landings of local species or the declines in CPUE shown in Fig. 2.

New trawl surveys are currently being conducted along the Israeli coast. The present study serves as an important spatial and temporal baseline for a detailed comparison of the status of fish and fisheries over the last two decades in the dynamic, quickly evolving habitats of the Mediterranean.

\section{ACKNOWLEDGEMENTS}

The research was partially funded by a grant from the Israeli Ministry of Agriculture and Rural Development to E. Spanier and O. Sonin and the Maurice Hatter Grant for Maritime Studies to D. Edelist. We also wish to thank the editor and reviewers, as well as Mr. Jonathan Liberzon for their useful comments, which greatly improved this article.

\section{REFERENCES}

Alsayes, A., S. Fattouh and S. Abu-Enin. - 2009. By-catch and discarding of trawl fisheries at the Mediterranean coast of Egypt. World J. Fish. Mar. Sci., 1: 199-205

Alverson, D.L., M.H. Freeberg. S.A. Murawski and J.G. Pope. 1994. A global assessment of fisheries bycatch and discards. FAO Fish. Tech. Paper, 339: 233.

Ben-Tuvia, A. - 1973. Man-made changes in the eastern Mediterranean Sea and their effect on the fishery resources. Mar. Biol., 19: 197-203.

Ben-Yami, M. - 1955. 1954 - 1955: over-fishing or bad season? Fisher. Bull., Haifa 6: 10-14 [in Hebrew]

Clarke, K.R. and R.M. Warwick. - 2001. Change in marine communities: an approach to statistical analysis and interpretation, $2^{\text {nd }}$ edition. PRIMER-E: Plymouth

Davies, R.W.D., S.J. Cripps, A. Nickson and G. Porter. - 2009. Defining and estimating global marine fisheries bycatch. Mar. Policy, 33: 661-672.

Demestre, M.S. de Juan, P. Sartor and A. Ligas. - 2008. Seasonal 
closures as a measure of trawling effort control in two Mediterranean trawling grounds: Effects on epibenthic communities. Mar. Pollut. Bull., 56: 1765-1773.

D'Onghia G., R. Carlucci, P. Maiorano and M. Panza. - 2003. Discards from deep-water bottom trawling in the eastern-central Mediterranean Sea and effects of mesh size changes. J. Northwest Atl. Fish. Soc., 31: 245-261.

El-Mor, M., J.S. El-Etreby. S. Mohammad and M.R. Sapota. 2002. A study on trash catch of the bottom trawl along Port-Said coast, Egypt. Oceanolog. Stud., 31: 45-55.

Esmeralda Costa, M., K. Erzini and T. Cerveira Borges. - 2008. Bycatch of crustacean and fish bottom trawl fisheries from southern Portugal (Algarve). Sci. Mar., 72(4): 801-814.

Galil, B.S. - 2007. Seeing red: alien species along the Mediterranean coast of Israel. Aquat. Invas., 2: 281-312.

Garcia, S. and A. Demetropoulos.- 1986. Management of Cyprus fisheries. FAO Fisheries Technical Paper, (250): 40 pp.

Golani, D. - 1993. The sandy shore of the Red Sea - launching pad for Lessepsian (Suez Canal) migrant fish colonizers of the eastern Mediterranean. J. Biogeog., 20(6): 579-585.

Golani, D., R. Reef-Motro, S. Ekshtein, A. Baranes and A. Diamant. - 2007. Ichthyofauna of the rocky coastal littoral of the Israeli Mediterranean, with reference to the paucity of Red Sea (Lessepsian) migrants in this habitat. Mar. Biol. Res., 3: 333-341.

Hall, M.A., D. L. Alverson and K. I. Metuzals. - 2000. By-Catch: problems and solutions. Mar. Pollut. Bull., 41(1-6): 204-219.

Jackson, J.B.C., M.X. Kirby, W.H. Berger, K.A. Bjorndal, L.W. Botsford, B.J. Bourque, R.H. Bradbury, R. Cooke, J. Erlandson, J.A. Estes, T.P. Hughes, S. Kidwell, C.B. Lange, H.S. Lenihan, J.M. Pandolfi, C.H. Peterson, R.S. Steneck, M.J. Tegner and R.R. Warner. - 2001. Historical overfishing and the recent collapse of coastal ecosystems. Science, 293: 629-637.

Kelleher, K. - 2005. Discards in the world's marine fisheries: an update. Rome: Food and Agriculture Organization of the United Nations, FAO; 2005. $131 \mathrm{pp}$

Lleonart, J. and F. Maynou. - 2003. Fish stock assessments in the Mediterranean: state of the art. Sci. Mar., 67(Suppl. 1): 37-49.

Machias A., V. Vassilopoulou, D. Vatsos, P. Bekas, A. Kallianiotis, C. Papaconstantinou and N. Tsimenides. - 2001. Bottom trawl discards in the northeastern Mediterranean Sea. Fish. Res., 53: 181-195.

Pauli, D., V. Christensen, S. Guénette, T.J. Pitcher, U.R. Sumaila, C.J. Walters, R. Watson and D. Zeller. - 2002. Towards sustainability in world fisheries. Nature, 418: 689-695.

Pisanty, S., O. Sonin and A. Alperovic. - 2000. Assessment of the influence of a summer trawling ban on the catch of the fishery. Technical Paper (in Hebrew). State of Israel, Ministry of Agriculture and Rural Development, Department of Fisheries. 17 pp.

Por, F.D. - 1989. The legacy of Tethys - an aquatic biogeography of the Levant. Dordrech, Kluwer 216 pp.

Rilov, G. and B.S. Galil. - 2009. Marine bioinvasions in the Mediterranean Sea - history, distribution and ecology. In: G. Rilov and J.A. Crooks (eds.), Biological invasions in marine ecosystems: ecological, management, and geographic perspectives, 3-11 pp. Springer-Verlag, Heidelberg.

Sacchi, J. 2008. The use of trawling nets in the Mediterranean. Problems and selectivity options. Options Mediterraneennes, B62: 87-96.

Sánchez P., M. Demestre and P. Martìn. - 2004. Characterization of the discards generated by bottom trawling in the northwestern Mediterranean. Fish. Res., 67: 71-80.

Sánchez P., P. Sartor, L. Recasens, A. Ligas, J. Martin, S. de Ranieri and M. Demestre. -2007 . Trawl catch composition during different fishing intensity periods in two Mediterranean demersal fishing grounds. Sci. Mar., 71(4): 765-773.

Sandler, A. and B. Herut. - 2000. Composition of clays along the continental shelf off Israel: contribution of the Nile versus local sources. Mar. Geol., 167: 339-354.

Scheinin, A.P. - 2010. The population of bottlenose dolphins (Tursiops truncatus), bottom trawl catch trends and the interaction between the two along the Mediterranean continental shelf of Israel. Ph.D. dissertation, Univ. Haifa, Israel.

Snovsky, G. and J. Shapiro - 1997. The fishery and aquaculture of Israel 1996 in figures. The state of Israel, Ministry of Agriculture - Department of Fisheries. 37pp.

Sonin O., E. Spanier, D. Levi, B. Patti, P. Rizzo and M.G. Andreoli. - 2007. Nanism (dwarfism) in fish: a comparison between red mullet Mullus barbatus from the southeastern and the central Mediterranean. Mar. Ecol. Prog. Ser., 343: 221-228.

Soykan H. and T. Kinacigil. - 2006. Bycatch associated with shrimp trawling in the Eastern Mediterranean (Taşucu Bay) In: Report of the ICES-FAO Working Group on Fishing Technology and Fish Behaviour (WGFTFB). Izmir, Turkey. 16.3: 50p.

Spanier E. and B.S. Galil. - 1991. Lessepsian migration a continuous biogeographical process. Endeavour, 15 (3): 102-106.

Stergiou, K.I., E. Christou and G. Petrakis. - 1997. Modelling and forecasting monthly fisheries catches: comparison of regression, univariate and multivariate time series methods. Fish. Res., 29: 55-95.

Sweeting C.J., F. Badalamenti, G. D'Anna, C. Pipitone and N.V. C. Polunin. - 2009. Steeper biomass spectra of demersal fish communities after trawler exclusion in Sicily. ICES. J. Mar. Sci., 66: 195-202.

Tsagarakis K., A. Machias, M. Giannoulaki, S. Somarakis and I. Karakassis. - 2008. Seasonal and temporal trends in metrics of fish community for otter-trawl discards in a Mediterranean ecosystem. ICES. J. Mar. Sci., 65(4): 539-550.

Wassenberg T.J., S. J. M. Blaber, C. Y. Burridge, D. T. Brewer, J. P. Salini and N. Grible. - 1997. The effectiveness of fish and shrimp trawls for the sampling of fish communities in tropical Australia. Fish. Res., 30: 241-251.

Scient. ed.: P. Martin.

Received May 14, 2010. Accepted February 14, 2011.

Published online July 13, 2011. 